\title{
Amount of practice and degree of attentional control have no influence on the adverse effect of alcohol in word categorization and visual search tasks
}

\author{
E. A. MAYLOR and P. M. A. RABBITT \\ Age and Cognitive Performance Research Centre, University of Manchester, Manchester, England
}

\begin{abstract}
This study investigated ways in which practice, and the closely related factor of automaticity, might modify the effects of alcohol $(0.8 \mathrm{ml} / \mathrm{kg}$ body weight) on word categorization and visual search. There were several possibilities: (1) Alcohol might have less effect on practiced than on unpracticed tasks, (2) practice with alcohol might allow adaptation to its effects, (3) alcohol might alter the rate of learning, and (4) alcohol might have less effect on tasks requiring automatic, as opposed to controlled, processing. Subjects participated in one session each day for either 2 or 5 days. The results revealed that alcohol impaired unpracticed and practiced performance to the same extent. In addition, those who practiced with alcohol for 4 days improved when switched to no alcohol on Day 5. Although alcohol impaired performance, there was no evidence that it reduced the benefit obtained from practice, that is, the rate of learning. Finally, almost identical effects of alcohol were observed for consistently mapped and variably mapped conditions (as developed by Schneider \& Shiffrin, 1977, to illustrate automatic and controlled processing, respectively). The four possibilities can therefore be answered as follows: Several days of practice at a task does not reduce the impairment caused by alcohol. There is no evidence for either statespecific practice or different rates of learning with and without alcohol. The effect of alcohol is not influenced by the degree of involvement of attentional control in a task.
\end{abstract}

Consider the following reasons why performance of a task may be more or less sensitive to alcohol effects: First, extended practice may minimize the influence of alcohol. Second, such practice may be more useful if obtained under alcohol-for example, it may be possible to learn to compensate for its effects-or, alternatively, alcohol may reduce the benefit obtained from practice so that it may take longer to attain levels of automaticity at which drug and stress effects are minimized (Fisk \& Schneider, 1982). Third, task difficulty may be a significant factor such that attentionally demanding tasks may remain vulnerable to alcohol effects at levels of practice at which less demanding tasks have gained immunity.

The literature on practice effects is inconclusive. Lewis, Dustman, and Beck (1969) examined the effect of alcohol on four cognitive and motor tasks by testing subjects after six weekly sessions without alcohol. On Sessions 7, 8 , and 9, subjects received no alcohol, a low dose of alcohol, or a medium dose in a randomized sequence. Performance on most of the tasks was not reliably altered

This research was supported by the Medical Research Council of Great Britain (Grant G221479N). The authors are grateful to Alan Kingstone for useful discussion and to John Cerella, Lester Kneger, and an anonymous referee for helpful comments on an earlier version of the paper. Requests for reprints should be addressed to Elizabeth Maylor at the Age and Cognitive Performance Research Centre, Oxford Road, Manchester M13 9PL, England. by either dose of alcohol, and the authors concluded that the "extended practice given before administration of alcohol could have stabilized test performance" (p. 629). However, few details of data scoring and analysis were given, and it would certainly be premature to claim that this study demonstrates the benefit of practice in reducing the influence of alcohol. The tasks and measurements chosen may have been insensitive to the effects of alcohol both early and late in practice.

It is not obvious whether or not practice under alcohol reduces alcohol effects. On the one hand, there may be a state-specific practice effect such that practice obtained in a particular state is more effective when the task is subsequently performed in that state than when performed in a different state. Also, the influence of alcohol may be reduced by practice if this results in learning to compensate for its effects or in the development of tolerance (reduced disruption in performance by a given drug dose when it is repeatedly administered). On the other hand, alcohol may decrease the benefit to be derived from practice by slowing the rate of learning. Thus, it would be advantageous to practice while sober, since a higher level of performance could be attained prior to the administration of alcohol. The evidence relating to these suggestions is far from conclusive. As noted by Poulos, Wolff, Zilm, Kaplan, and Cappell (1981), there are very few investigations of tolerance to alcohol in humans, particularly with respect to effects on cognitive functioning. 
In relation to the third question, Norman (1976) noted that "automatic, non-conscious actions" should be "less susceptible to disruption" (p. 65) by high levels of arousal. Hasher and Zacks (1979) suggested that if increased arousal reduces available cognitive capacity (Kahneman, 1973), automatic operations, which require little capacity, should function as normal whereas effortful, capacity-demanding processes should be impaired. Some support for this idea that automatic performance is relatively resistant to reduction of cognitive capacity comes from Fisk and Schneider (1982), who examined the effects of alcohol on automatic and effortful processing. Subjects were tested with and without alcohol on two visual search tasks. For the task involving automatic processing, alcohol resulted in a drop in detection performance of $2.2 \%$; the impairment for the task involving effortful processing was $9.6 \%$. Thus, it would appear that tasks in which it is possible to attain "automatic" performance are less sensitive to alcohol than are those in which attention is subject to control. It should be noted that automaticity, as an empirical construct, is defined in two ways. One definition is dependent on level of practice, since automatic performance can be attained only after considerable experience of a task. The other definition is independent of practice, since only tasks in which attention need never be shifted voluntarily during performance can become automatic, whereas tasks in which attention has to be shifted consciously and refocused by continually changing task demands can never become so. In investigating the possible interactions of alcohol with degree of automaticity, these distinct factors of level of practice and task type must be separated.

\section{The Present Study}

These questions were investigated in the present study by adopting the following design (see Table 1). Two groups of subjects participated in two sessions and two further groups participated in five sessions. One of the two-session groups received no alcohol in the first session and alcohol in the second (NA/A); this was reversed for the other two-session group (A/NA). The five-session groups were similar except that they remained in the same conditions for the first four sessions and then reversed conditions on the fifth session (NA-- $/ \mathrm{A}$ and A - - - /NA). The subjects performed the same tasks in the same order in each session. This design allowed several comparisons to be made. The effect of alcohol on relatively unpracticed performance could be compared with that on more practiced performance. From a comparison of the five-session groups over the first four sessions, it would be possible to discover whether or not subjects could, in any sense, adapt to, or learn to compensate for, the effect of alcohol. In other words, is the effect of alcohol as large in the fourth session as in the first? The final issue was addressed by comparing the effect of alcohol on processing tasks well separated along the automatic-effortful continuum. (It should be noted that the "automatic" tasks may not have involved purely automatic processing as defined by strict criteria for automaticity; see, e.g., Posner, 1978. Nevertheless, it is certain that the tasks of interest, while possibly not at the extremes of the continuum, did involve very different degrees of attentional control.) The present paper reports two of the tasks chosen for investigation and these will now be described.

\section{Word Categorization}

Word categorization was based on a task designed by Schneider and Fisk (1984). Subjects were first presented with a category name and then asked to respond as quickly as possible (by pressing one of three keys) according to the location of the target (a member of that category) among two distractors (members of other categories). They distinguished between two conditions based on the relationship between the target and distractor set and the amount of practice. According to Schneider and Shiffrin's (1977) model, performance on a varied mapping (VM) task can never become automatic, since subjects cannot consistently attend to the same stimuli on all trials but must consciously refocus their attention to deal with each new event. A stimulus that was a target on one trial might be a distractor on the next trial. Readjustment to these continual changes requires effortful or controlled processing. Practice can improve overall performance, but cannot alter this high level of attentional demand. In contrast, for a consistent mapping (CM) task, the same stimuli always occur as targets and never as distractors. The initial adjustment to task demands requires effortful control of attention. As practice progresses, performance becomes increasingly automatic. Thus, Hasher and Zack's (1979) hypothesis would predict that reduction of cognitive capacity by alcohol should affect performance on VM and CM tasks to approximately the same extent early in practice, since, at this stage, both require effortful control of attention. However, as practice progresses, the effects of alcohol should become increasingly differentiated between tasks. VM tasks, in which control has to be exercised, should be affected more than CM tasks, in which automaticity is increasingly attained. Another interesting point concerns the effect of alcohol on the rate at which practice shifts performance on the CM task from an effortful toward an automatic mode of processing. This is indexed by the rate of divergence of performance in the $\mathrm{CM}$ and VM tasks with practice, and can therefore be compared with and without alcohol.

\section{Visual Search}

This task was adapted from Experiment 2 of Schneider and Shiffrin (1977). Again, CM and VM conditions were contrasted, but, unlike word categorization, they were well separated along the automatic-effortful continuum from the start of the task. This was achieved by making use of an already well-learned categorical difference between targets and distractors in the $\mathrm{CM}$ conditions (that 
is, letters vs. digits). For example, some subjects were shown a memory set of either digits (CM) or letters (VM) and were then required to search for these items among letters. In this way, any differences in the effect of alcohol on CM and VM early in practice could be compared with those observed late in practice.

\section{METHOD}

\section{Subjects}

Eighty volunteers ( 40 male, 40 female) were recruited, through advertisements placed around the University of Manchester. They were all light or moderate social drinkers. Half of the subjects agreed to participate in the 2-day experiment, which required approximately $1 \mathrm{~h}$ at the same time on each of 2 consecutive days. The remaining subjects came at the same time for $1 \mathrm{~h}$ every day from Monday to Friday. The 2-day subjects received $£ 5$ on completion of the experiment; those who participated for a week were given $£ 10$ plus a punctuality bonus of up to $£ 5$ ( $£ 1$ for each day on which they arrived not more than $10 \mathrm{~min}$ late). Further details of the subjects are summarized in Table 1.

\section{Apparatus and Stimuli}

Blood alcohol concentration was estimated to the nearest $5 \mathrm{mg}$ alcohol/100 ml blood using a Lion Alcolmeter S-D2 (Breathalyzer) obtained from Lion Laboratories, Barry, U.K. A BBC “'B" microcomputer was used for both tasks to control stimulus presentation, record responses (to the nearest centisecond), and store and analyze data.

\section{Word Categorization}

Words were presented in white upper case at the center of an RGB medium-resolution color monitor. Fach letter was $5 \mathrm{~mm}$ wide and $6 \mathrm{~mm}$ high. When three words were presented simultaneously, the vertical separation between the rows was $10 \mathrm{~mm}$. The response keys were " $M$," " $K$," and " $O$ ' for right-handed subjects, and " $C$," " $D$," and " $W$ " for (approximately 3) left-handed subjects. These were operated with the first, second, and third fingers of the dominant hand and corresponded to responses of "bottom," " middle," and "top" lines. The subjects were seated with their eyes level with, and approximately $400 \mathrm{~mm}$ away from, the center of the screen.

Two sets of four semantic categories were prepared. Set A comprised animals, body parts, colors, and weather; set B comprised clothing, weapons, furniture, and trees. For each category, the four members chosen (1) contained between three and five letters and (2) ranked in dominance between 1 and 12 on the Battig and Montague (1969) norms.

\section{Visual Search}

Letters and digits were presented in the center of the screen, each character measuring $5 \times 6 \mathrm{~mm}$, as for word categorization. The horizontal separation between letters was $5 \mathrm{~mm}$ and the vertical sepa-

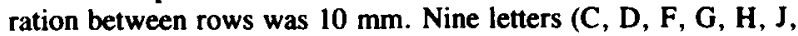
$K, L$, and $M)$ and nine digits $(1,2,3,4,5,6,7,8$, and 9) were used as the stimuli. The response keys, operated by the left and right index fingers, were " $Z$ "' for "target present" responses and " $\%$ " for "target absent" responses.

\section{Design and Procedure}

Most of the subjects were tested in pairs on identical systems separated by screens in the same laboratory. The remaining subjects were tested individually. All were asked not to eat for at least $2 \mathrm{~h}$ before each session (unless they were booked to begin at $0900 \mathrm{~h}$, in which case they were encouraged to eat breakfast as early as practically possible). Before the experiment, each subject was weighed and asked to sign a consent form agreeing to take part in the study.

Each session began with the subject's drinking $500 \mathrm{ml}$ of fluid: $150 \mathrm{ml}$ concentrated orange drink mixed with $0.8 \mathrm{ml}$ alcohol in the form of vodka (37.5\% alcohol by volume) per $\mathrm{kg}$ body weight and water, for the alcohol sessions, and $150 \mathrm{ml}$ orange drink mixed with $350 \mathrm{ml}$ water, with a few drops of vodka floated on the top, for the no-alcohol sessions. The subject was instructed to finish the drink within $5 \mathrm{~min}$, and was not provided with any information regarding the quantity of alcohol in the drink.

There were 20 subjects in each of the four conditions (NA/A, A/NA, NA $---/ A$, and A $---/$ NA), as summarized in Table 1. (The unequal numbers of male and female subjects in the two 5 day conditions were due to an administrative error.)

The procedure for each session was identical in all conditions. When the drink was finished, the subject threw 60 darts at a target. This was included principally to occupy the subjects for approximately $5 \mathrm{~min}$ while some alcohol was absorbed into their blood before they began the experimental tasks (although the subjects were not informed of this). Word categorization, which took approximately $15 \mathrm{~min}$, was immediately followed by the first breath reading. After the subjects had been occupied for a further $15 \mathrm{~min}$ by a simple computer game (see Maylor \& Rabbitt, 1987b), a second breath reading was taken before the visual search task (15 min) was carried out. Before the subjects left, a third breath reading was taken. Each session was completed in approximately $1 \mathrm{~h}$.

\section{Word Categorization}

In each session, there were 200 trials of word categorization. These were divided into eight blocks with an opportunity to pause between blocks signaled by the screen instruction "Press spacebar to continue when ready."

Table 1

Subject Details

\begin{tabular}{|c|c|c|c|c|c|c|c|c|}
\hline \multirow[b]{2}{*}{ Condition } & \multicolumn{2}{|c|}{$\begin{array}{l}\text { Number of } \\
\text { Subjects }\end{array}$} & \multicolumn{2}{|c|}{ Age (years) } & \multicolumn{2}{|c|}{ Weight (kg) } & \multicolumn{2}{|c|}{$\begin{array}{c}\text { Number of } \\
\text { Subjects Tested }\end{array}$} \\
\hline & Males & Females & $M$ & $S D$ & $M$ & $S D$ & Before $1300 \mathrm{~h}$ & After $1300 \mathrm{~h}$ \\
\hline \multicolumn{9}{|c|}{ 2-Day } \\
\hline NA/A & 10 & 10 & 21.5 & 4.5 & 69.4 & 10.2 & 7 & 13 \\
\hline A/NA & 10 & 10 & 21.2 & 4.4 & 67.0 & 9.4 & 6 & 14 \\
\hline \multicolumn{9}{|c|}{ 5-Day } \\
\hline $\mathbf{N A}---/ \mathbf{A}$ & 11 & 9 & 20.5 & 1.5 & 69.1 & 10.7 & 5 & 15 \\
\hline $\mathbf{A}---/ \mathbf{N A}$ & 9 & 11 & 20.6 & 2.4 & 65.4 & 9.8 & 7 & 13 \\
\hline
\end{tabular}

Note-NA $=$ no alcohol, $\mathrm{A}=$ alcohol. 
On each trial, one of the eight categories (chosen randomly) was presented in the center of the screen for $1 \mathrm{sec}$. The subject was required to make a mental note of the category but to respond in no other way. There was then a blank interval of $0.5 \mathrm{sec}$ before three words appeared, from three separate categories, one above the other, with the middle word in the position previously occupied by the category name. These remained on the screen until the subject had responded by pressing one of the three response keys. A single target (that is, a member of the category) appeared on every trial accompanied by two distractors. The subject's task was to determine the target's location (top, middle, or bottom line, which was varied randomly from trial to trial) and to respond as quickly and as accurately as possible by pressing the appropriate key. Following the response, there was a blank interval of $1 \mathrm{sec}$ before the next trial was presented.

There were two conditions, with approximately equal numbers of trials in each condition within a session. Items in four of the eight categories appeared only as targets and never as distractors. Thus, for half of the categories, there was a CM (consistent mapping) between the target and distractor words so that whenever an item from a CM category appeared, it received a consistent response. For the other four categories, there was a VM (varied mapping), so that items in those categories were targets on some trials and distractors on others. Therefore, a subject could not consistently respond to the same stimuli on all trials in which they appeared. The subjects were not informed of the CM/VM distinction and appeared to be unaware of it even after 1,000 trials (although, as will be seen, it greatly affected their performance).

To control for possible differences between performance across the eight chosen semantic categories, half of the subjects in each of the four conditions shown in Table 1 were given set $\mathrm{A}$ as $\mathrm{CM}$ categories and set $B$ as VM categories; the others were given the reverse (that is, set $B$ for $C M$ and set $A$ for $V M$ ).

The data from each session were divided into two blocks of 100 trials. Since the word-categorization task was carried out during the period when blood alcohol concentration was probably changing (rising) most rapidly, only the results from the second block will be reported.

\section{Visual Search}

The visual search task was divided into two blocks, CM and VM, with 100 trials in each block. Half of the subjects in each of the four conditions shown in Table 1 performed the tasks in the order CM-VM in every session; the other subjects performed the tasks in the reverse order.

Each block was divided into four mini-blocks that enabled the subject to pause between them, at the instruction "Press spacebar to continue when ready." A trial began with the presentation of two items (either both letters or both digits, depending on the condition) in the center of the screen (the memory set). These remained on the screen until the subject pressed the spacebar to indicate that they had been memorized. It was made clear that this part of the task was not timed. The memory set was then removed and replaced, after a blank interval of $1 \mathrm{sec}$, by the search set of four items. These appeared immediately above and below the position previously occupied by the memory set and, depending on the condition, were all digits, all letters, or both digits and letters. The subject's task was to decide as quickly as possible whether one of the items in the memory set was present in the search set ("target present") or not ("target absent") and to respond by pressing the appropriate key. (There was never more than one target present.) Following the response, the screen was cleared and, after an interval of $1 \mathrm{sec}$, the next trial began with a new memory set. There were approximately equal numbers of target-present and target-absent trials. Every aspect of the procedure was randomized for each subject, and from trial to trial-for example, the spatial location of the target within the $2 \times 2$ search set.
The CM and VM conditions were similar in principle to those of the word-categorization experiment. Thus, half of the subjects in each of the four conditions shown in Table 1 were required to search for digits among letters (CM) in one block and for letters among letters in the other (VM); the other half searched for letters among digits (CM) and digits among digits (VM).

\section{RESULTS AND DISCUSSION}

\section{Blood Alcohol Concentration}

For the no-alcohol sessions, all the readings were zero. The mean blood alcohol concentration (BAC) estimates for the alcohol sessions were $59.1 \mathrm{mg}(S D=18.0)$, $63.0 \mathrm{mg}(S D=16.7)$, and $63.8 \mathrm{mg}(S D=15.6)$ alcohol $/ 100 \mathrm{ml}$ blood, approximately 20,40 , and $60 \mathrm{~min}$, respectively, after completion of the drink, corresponding to the end of the word-categorization task, the beginning of the visual search task, and the end of the visual search task. For the particular dose employed in the present study, BAC would probably have risen slightly and fallen again between the second and third readings (see Drew, Colquhoun, \& Long, 1959). Note that the legal BAC limit for driving in the U.K. is currently set at $80 \mathrm{mg} / 100 \mathrm{ml}$ blood.

\section{Word Categorization}

\section{Two-Day Experiment}

The data from the 2-day experiment are summarized in Figure 1. The median reaction times (correct responses only) and error rates were analyzed in two separate analyses of variance, with one between-subjects factor (alcohol group, either NA/A or A/NA), and two withinsubjects factors (day, 1 or 2 , and mapping condition, $\mathrm{CM}$ or VM).

For median reaction times, there was no effect of group $[F(1,38)=1.10, p>.1]$, but there were significant effects of day $[F(1,38)=10.99, p<.01]$ and mapping $[F(1,38)=40.27, p<.01]$. There was also an interaction between day and mapping $[F(1,38)=5.83$, $p<.05]$. The significant effects are all as expected from the results of Schneider and Fisk (1984). Thus, subjects responded faster overall on Day $2(846 \mathrm{msec})$ than on Day 1 (905 $\mathrm{msec})$, and faster in the CM (836 msec) than in the VM (916 msec) condition. The day $\times$ mapping interaction was due to an increase in the difference between CM and VM from $59 \mathrm{msec}$ on Day 1 to $101 \mathrm{msec}$ on Day 2. To summarize, subjects speeded up with practice. This improvement was greater for CM than for VM. Alcohol had no effect on reaction time.

There were no main effects in the analysis of errors, but there were significant interactions between alcohol group and day of practice $[F(1,38)=6.34, p<.05]$ and between day and mapping $[F(1,38)=4.93, p<.05]$. The latter interaction suggests that the larger decrease in reaction time across days for CM than for VM is actually associated with a larger increase in error rate, indicating an element of speed-error tradeoff. The former interaction is more important in the present context because 

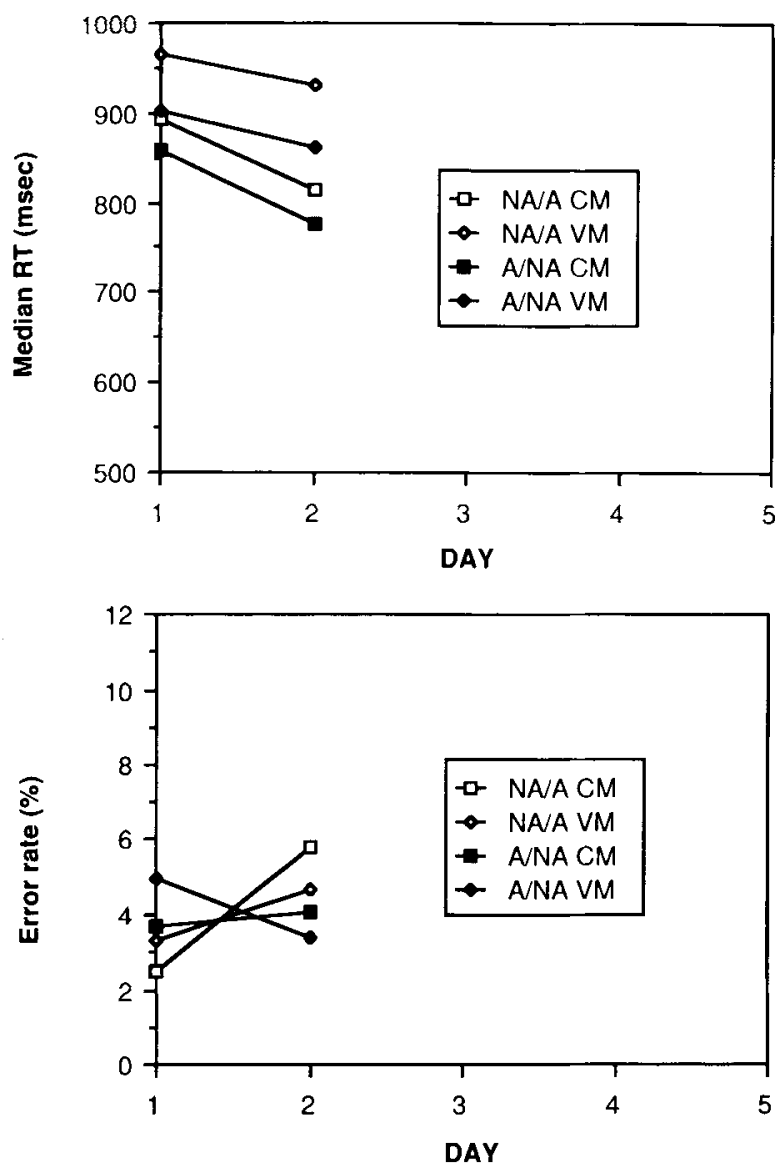

Figure 1. Overall means for the word categorization task (2-day experiment).

it indicates that alcohol significantly increased overall error rate by $1.475 \%$ (the average across the two groups). Not surprisingly, the effect of alcohol was similar for CM (up by $1.45 \%$ ) and VM (up by $1.5 \%$ ), as evidenced by the absence of a three-way interaction $(F<1)$.

Figure 1 shows that from Day 1 to Day 2, the increase in error rate of 2.35 for the NA/A group was larger than the decrease of 0.60 for the A/NA group. However, these results should be interpreted in the context of the data for Days 1 and 2 of the 5-day experiment (Figure 2), that is, the NA/NA and A/A conditions, in which there was also a tendency toward an overall increase in error rate of 1.20 from Day 1 to Day 2 (see also Table 2). It seems that there is a tendency over the first 2 days to adjust the speed-error tradeoff in the direction of increasing the number of errors. The effects of changing condition (NA/A and A/NA) groups) are superimposed on this trend. Release from alcohol on Day 2 (A/NA) results in a small overall reduction in errors, counteracting the trend with practice. Transfer to alcohol (NA/A) results in even more errors than are observed as a function of practice alone. It appears that the effects of practice and of alcohol on errors are independent in this task.
The results of the 2-day word-categorization experiment demonstrate that (1) alcohol has no significant effect on speed, and (2) alcohol increases error rate equally for CM and VM.

\section{Five-Day Experiment}

The analysis of the data from the 5-day experiment was divided into Days 1 to 4 (to investigate learning with and without alcohol) and Days 4 and 5 (to compare the effect of alcohol late in practice with that seen early in practice in the 2-day experiment).

Days 1 to 4 . The analysis conducted on the median reaction times for Days 1-4 was the same as that conducted for the 2-day experiment, except that the number of levels on the day factor was 4 rather than 2 . There was no effect of alcohol group $[F(1,38)=1.98, p>.1]$, but there were highly significant effects of day $[F(3,114)=$ 40.78, $p<.01]$ and mapping $[F(1,38)=76.96$, $p<.01]$. The only interaction was between day and mapping $[F(3,114)=2.69, p<.05]$. These results are very similar to those from the 2-day experiment, with alcohol again having no influence on response speed. Reaction time decreased across the 4 days (the day effect), with
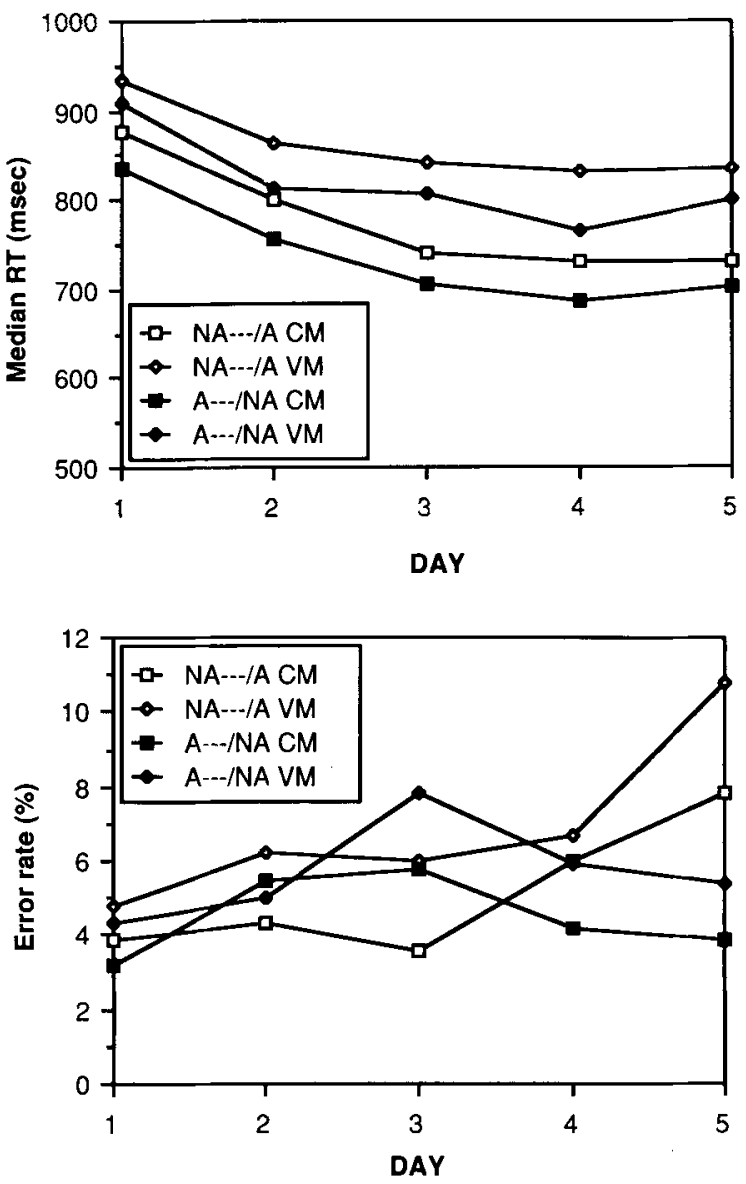

Figure 2. Overall means for the word categorization task (5-day experiment). 
the difference between $\mathrm{CM}$ and VM increasing across the 4 days from $64 \mathrm{msec}$ on Day 1 to $90 \mathrm{msec}$ on Day 4 (the day $\times$ mapping interaction). The absence of a three-way interaction $(F<1)$ indicates that alcohol has no influence on the improvement in speed with practice of $\mathrm{CM}$ with respect to $\mathrm{VM}$, and thus on the rate of change in processing for $\mathrm{CM}$ from effortful toward automatic.

A similar analysis for error rate revealed an effect of day that approached significance $[F(3,114)=2.44$, $p<.07]$ and a highly significant effect of mapping $[F(1,38)=9.91, p<.01]$. As can be seen from Figure 2, error rate increased from Day 1 to Day 4, with the linear trend reaching significance $[F(1,114)=5.71$, $p<.05]$. More errors were made in the VM $(5.84 \%)$ than in the CM $(4.56 \%)$ condition.

There was no overall effect of alcohol, which is surprising, since it significantly increased error rate for the 2-day groups. In several previous 2-day studies (e.g., Maylor \& Rabbitt, 1987a) in which half of the subjects received no alcohol on Day 1 and alcohol on Day 2 while the order was reversed for the remaining half, betweensubjects analyses on the data from Day 1 alone failed to reveal significant effects of alcohol. However, when both days' results were included in analyses similar to that used for the present 2-day experiment, highly significant effects of alcohol were found, such that the performance of the NA/A group was worse on Day 2 than on Day 1, whereas performance for the A/NA group improved. The problem, of course, is that the effects of such low doses of alcohol are small relative to the enormous variation between subjects (see also Huntley, 1973). There are at least two courses of action available to the investigator wishing to study alcohol effects (and these are not necessarily mutually exclusive). First, a preliminary screening task may be used in an attempt to select only subjects within a particular range of ability, or to match them according to ability across the two groups. The second is the method adopted here, that is, a counterbalanced design in which the emphasis is placed on the change in performance when the conditions are reversed; hence, the analyses of Days 4 and 5. The latter design is obviously obligatory when, as in this experiment, the aim is to study practice effects and to compare the effects of the imposition and removal of alcohol early and late in practice. Only by testing very large groups of volunteers, selecting and matching in terms of task-relevant criteria (which remain to be determined), can both suggestions be incorporated into an experimental design.

To summarize the results of Days 1 to 4 , there is no evidence that the rate of learning (in terms of the overall change in speed with practice) is altered by alcohol. Furthermore, it is clear that considerable learning occurs both with and without alcohol, such that the difference in speed between the CM and VM conditions increases with practice. In other words, alcohol does not affect the rate of acquisition of automaticity in the CM task. With or without alcohol, subjects are more accurate for $\mathrm{CM}$ than for VM.
Days 4 and 5. The only significant effect from the analysis of median reaction times from Days 4 and 5 was that of mapping condition $[F(1,38)=71.35, p<.01], \mathrm{CM}$ being 96 msec faster than VM.

For errors, there were significant effects of day $[F(1,38)$ $=4.58, p<.05]$ and mapping $[F(1,38)=12.80$, $p<.01$ ]. It can be seen from Figure 2 that more errors were made on Day 5 than on Day 4 and that CM was more accurate than VM. In addition, there was a highly significant interaction between alcohol group and day $[F(1,38)=7.90, p<.01]$. The overall differences in error rate from Day 4 to Day 5 (2.95 and -0.40 for the NA $---/ A$ and $A---/$ NA groups, respectively) should be considered in the light of the overall trend from Days 1 to 4 for error rate to increase across days. The absence of a three-way interaction indicates that alcohol has a similar effect on error rate for CM and VM, despite the fact that, by Days 4 and 5 , the two conditions are highly differentiated, $\mathrm{CM}$ being much faster and more accurate than VM. It is clear from a comparison of Tables 2 and 3 that the overall effect of alcohol is at least as large on Days 4 and 5 as on Days 1 and 2. This was confirmed by a further analysis of variance with length of experiment ( 2 days or 5 days) and alcohol group (NA or A on Day 1/Day 4, and A or NA on Day 2/Day 5) as betweensubjects factors and day (1/4 or 2/5) and mapping condition as within-subjects factors. The alcohol group $\times$ day interaction was, of course, highly significant $[F(1,76)=$ $14.21, p<.01$ ], but there was no interaction between length of experiment, alcohol group, and day $(F<1)$.

Three main conclusions can be drawn from this wordcategorization experiment. First, concerning speed, alcohol has no effect on the rate of learning, in terms of both the overall improvement across days and the specific improvement with practice of CM with respect to VM. Second, alcohol impairs accuracy, and to the same extent in CM and VM conditions. This, of course, was expected early in practice, when performance in both tasks

Table 2

Overall Increases in Error Rate from Day 1 to Day 2 (2-Day and 5-Day Experiments)

Word Categorization

Day 2

$\begin{array}{cccc} & & \text { NA } & \text { A } \\ \text { Day 1 } & \text { NA } & 0.90 & 2.35 \\ & \text { A } & -0.60 & 1.50\end{array}$

Visual Search

Day 2

$\begin{array}{cccc} & & \text { NA } & \text { A } \\ \text { Day } 1 & \text { NA } & 0.88 & 1.97 \\ & \text { A } & -1.50 & 0.85\end{array}$


Table 3

Overall Increases in Error Rate from Day 4 to Day 5 (5-Day Experiment Only)

Word Categorization

Day 5

$\begin{array}{cccc} & & \text { NA } & \text { A } \\ & \text { NA } & - & 2.95 \\ & \text { A } 4 & -0.40 & -\end{array}$

Visual Search

Day 5

NA A

NA

2.20

Day 4

A $\quad-1.65$

demanded effortful control. It is more surprising (being inconsistent with Hasher \& Zack's, 1979, hypothesis) to find that late in practice, the effects of alcohol remain equivalent in the two conditions, since the CM task, in contrast to the VM task, is performed with an increasing degree of automaticity. Third, the effect of alcohol on error rate is not reduced by practice at the task.

\section{Visual Search}

\section{Two-Day Experiment}

The median reaction times (for correct responses only) were analyzed with alcohol group (NA/A or A/NA) as the between-subjects factor and day ( 1 or 2 ), mapping condition (CM or VM), and target presence (target present [TP] or target absent [TA]) as within-subjects factors. There were highly significant effects of day $[F(1,38)=$ $106.01, p<.01]$, mapping condition $[F(1,38)=154.65$, $p<.01]$, and target presence $[F(1,38)=99.36$, $p<.01]$. In addition, there were interactions between day and mapping condition $[F(1,38)=7.81, p<.01]$, day and target presence $[F(1,38)=16.66, p<.01]$, and mapping condition and target presence $[F(1,38)=13.56$, $p<.01]$. The main effects simply indicate that subjects were faster on Day 2 than on Day 1, faster for CM than for VM, and faster to respond when a target was present $(608 \mathrm{msec})$ than when it was absent $(743 \mathrm{msec})$. The day $x$ mapping interaction can be seen, from Figure 3, to result from the greater improvement in performance from Day 1 to Day 2 for VM than for CM. This was to be expected, since the CM condition was probably approaching automaticity at the start and therefore was much closer to the theoretical system asymptote for this task. The difference between TP and TA was larger on Day 1 $(157 \mathrm{msec})$ than on Day $2(114 \mathrm{msec})$, leading to the day $x$ target presence interaction. Finally, the mapping condition $\times$ target presence interaction, which has been well documented by Schneider and Shiffrin (1977), was found in this experiment, that is, the TP-TA difference was substantially larger for VM $(216 \mathrm{msec})$ than for CM $(55 \mathrm{msec})$ trials. However, the important result for the present study is that, as for word categorization, there was no effect of alcohol group and no interactions involving alcohol. Thus, alcohol again appears to have no influence on response speed.

For errors, there were no significant effects of alcohol group or day (both $F \mathbf{S}<1$ ), but there were highly significant effects of mapping condition $[F(1,38)=43.81$, $p<.01]$ and target presence $[F(1,38)=62.51$, $p<.01]$. There were significant interactions between alcohol group and day $[F(1,38)=12.97, p<.01]$ and between mapping condition and target presence $[F(1,38)=$ $12.59, p<.01]$. Thus, subjects were more accurate for CM than for VM and more accurate for TP than for TA, the difference between TP and TA being larger for VM (2.34) than for CM (1.23). The presence of an alcohol group $\times$ day interaction and the absence of a three-way interaction between alcohol group, day, and mapping condition $[F(1,38)=1.39, p>.05]$ are most important here because they indicate that alcohol affects accuracy equally for $C M$ and $V M$. Figure 3 shows an almost perfect cross-
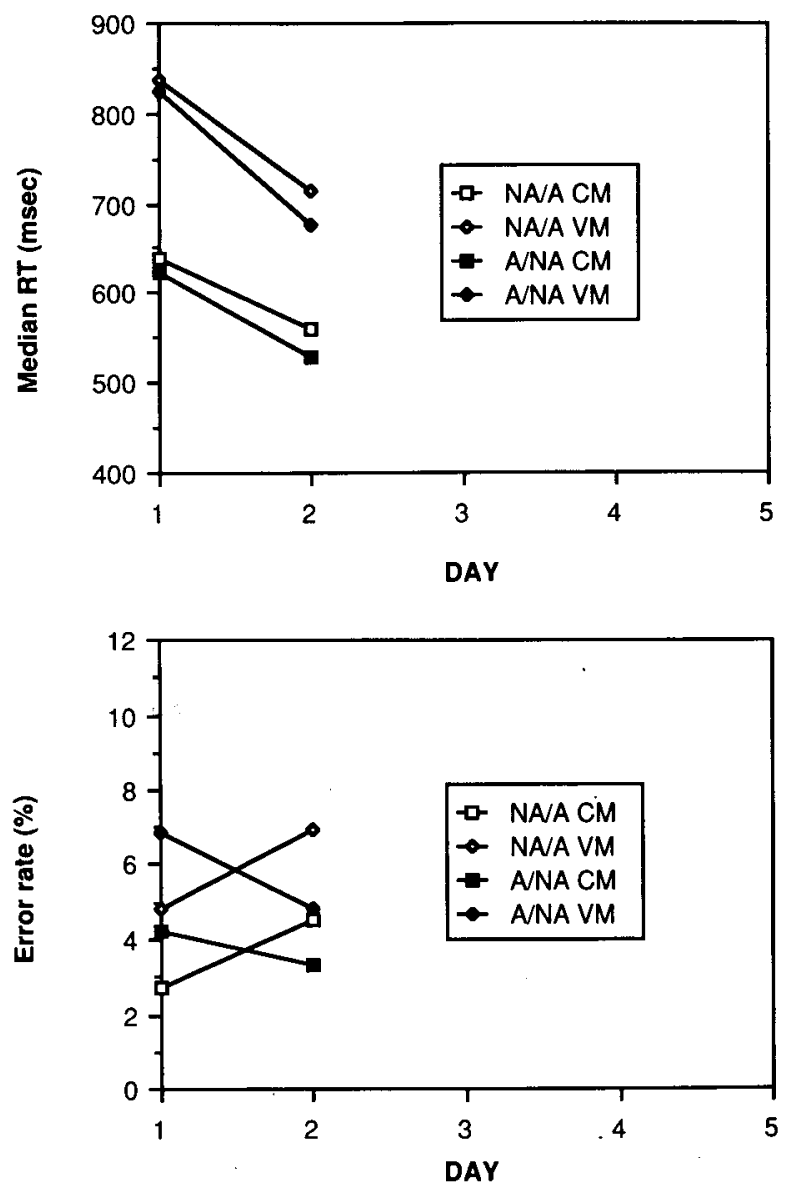

Figure 3. Overall means for the visual search task (2-day experiment). 
over from Day 1 to Day 2, the overall effect of alcohol being to increase error rate by 1.74 (the average difference between the no-alcohol and alcohol conditions).

The first conclusion to be drawn from this 2-day visual search experiment is that the mapping-condition manipulation (making use of the already well-learned letter and digit categories) was successful early in practice in producing faster and more accurate performance for $\mathrm{CM}$ than for VM. Second, alcohol had no effect on response speed. Third, subjects made more errors with alcohol, the increase being almost identical for CM and VM. In relation to this last point, it is interesting to note that the effect of alcohol on error rate was similar for TP and TA trials despite the fact that TP trials were more automatically processed in the empirical sense of being both faster and more accurate than TA trials.

\section{Five-Day Experiment}

Days 1 to 4 . The analysis of median reaction times revealed effects of day $[F(3,114)=132.96, p<.01]$, mapping condition $[F(1,38)=79.93, p<.01]$, and target presence $[F(1,38)=85.87, p<.01]$, in addition to interactions between day and mapping condition $[F(3,114)$ $=18.45, p<.01]$, day and target presence $[F(3,114)$ $=18.97, p<.01]$, mapping condition and target presence $[F(1,38)=33.94, p<.01]$, and day, mapping condition, and target prescnce $[F(3,114)=16.94$, $p<.01]$. The main effects and two-way interactions were all as described for the 2-day experiment. The threeway interaction reflects a larger reduction across days in the difference between TP and TA for VM (231 and $93 \mathrm{msec}$ on Days 1 and 4, respectively) than for CM (60 and $41 \mathrm{msec}$ ), a trend that was present for the 2-day experiment but insignificant $[F(1,38)=2.61, p>.05]$. Again, alcohol had no influence on reaction time.

For error rates, there was no effect of alcohol group $(F<1)$, but there were significant effects of day $[F(3,114)=5.98, p<.01]$, mapping condition $[F(1,38)$ $=42.88, p<.01]$, and target presence $[F(1,38)=$ $66.58, p<.01]$. There was also an interaction between mapping condition and target presence $[F(1,38)=64.23$, $p<.01$, which was very similar to that observed in the 2-day experiment.

The absence of an overall alcohol group effect is, at first, puzzling, since an effect of alcohol on error rate was clearly present for the 2-day experiment. A similar observation for word categorization led to a discussion of the problem of between-subjects analyses with respect to alcohol. In fact, it appears that while the subjects in the two groups of the 2-day experiment were approximately equivalent in terms of overall accuracy, the 5-day groups were not. Thus, for example, in the present task, subjects in the NA/A and NA - - - /A groups made approximately equal numbers of errors on Day 1 , while the A - - - /NA group made rather fewer errors than did the A/NA group. Also, when the conditions were reversed on Day 2 of the 2-day experiment, the NA/A group performed with approximately the same accuracy as the
A/NA group on Day 1, and vice versa, whereas, as can be seen from Figure 4, the A-- - /NA group was more accurate on Day 5 than was the NA - - - /A group on Day 4, and the NA - - - /A group on Day 5 made more errors than did the A-- -/NA group on Day 4. It is therefore concluded that the absence of an effect of alcohol on error rate for Days 1 to 4 is partly attributable to a group difference such that the subjects given alcohol in the 5-day experiment were generally more accurate than those given no alcohol. However, this unfortunate absence of perfect matching does not affect the important comparisons of interest (see Tables 2 and 3), and thus the main conclusions drawn from these data.

The day effect had both a linear $[F(1,38)=10.58$, $p<.01]$ and a cubic component $[F(1,38)=7.33$, $p<.05$ ], the means for Days 1 to 4 being 4.44, 5.30, 4.79 , and 5.75. Thus, for both visual search and word categorization, the decrease in reaction time across days was accompanied by an increase in error rate. This was less dramatic for the present visual search task and, in fact, included a reversal for Days 2 and 3 for which there appears to be no obvious explanation.

The mapping-condition and target-presence effects were as expected, and since the former effect did not interact
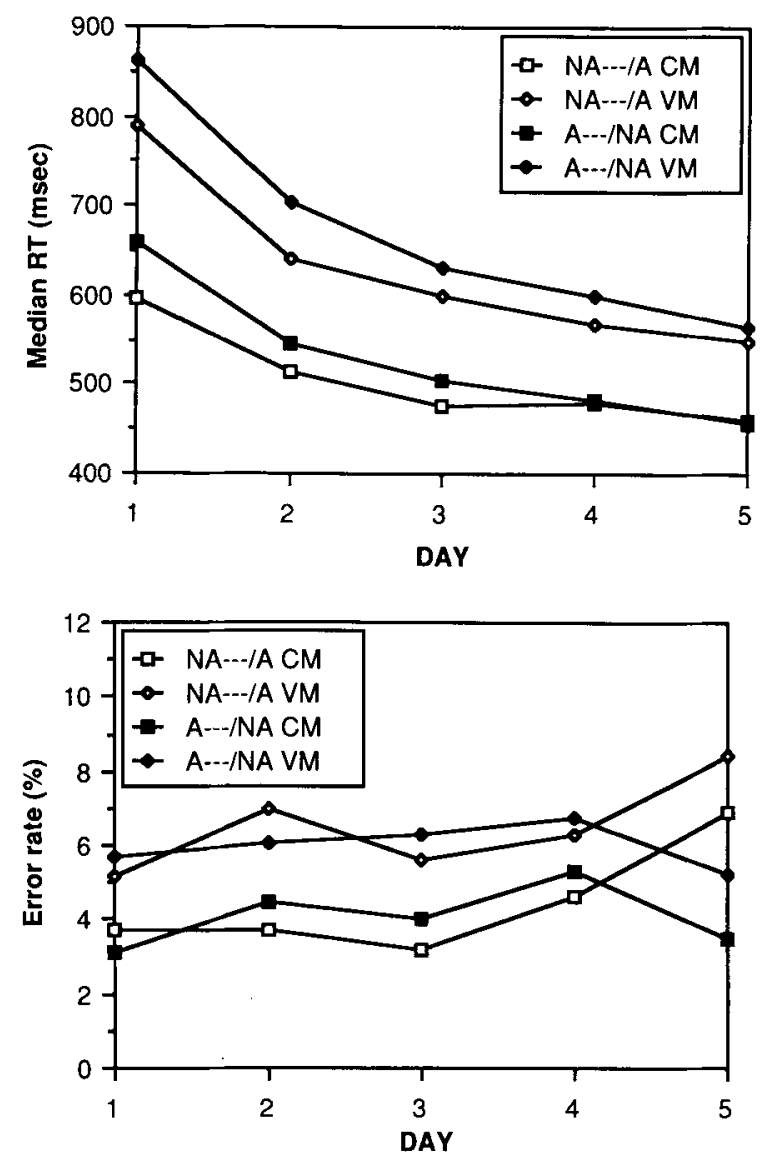

Figure 4. Overall means for the visual search task (5-day experiment). 
with day it appears that, at least in terms of accuracy, there is a stable $\mathrm{CM} / \mathrm{VM}$ difference that can be used to compare the effect of reversing the alcohol conditions after 4 days of practice with that observed after only 1 day of practice.

Days 4 and 5. The results for median reaction times were as for Days 1 to 4, except that there were no interactions involving day. There were significant effects of day $[F(1,38)=38.01, p<.01]$, mapping condition $[F(1,38)=56.55, p<.01]$, and target presence $[F(1,38)$ $=42.15, p<.01]$, and an interaction between mapping condition and target presence $[F(1,38)=11.70$, $p<.01]$.

For errors, there were the usual effects of mapping condition $[F(1,38)=9.82, p<.01]$ and target presence $[F(1,38)=20.02, p<.01]$, and an interaction between them $[F(1,38)=32.90, p<.01]$. However, the important result was a highly significant interaction between alcohol group and day $[F(1,38)=24.05, p<.01]$. There were no significant three- or four-way interactions (all Fs $<1$ ), indicating that the effect of alcohol on error rate was independent of both mapping condition and target presence. It can be seen from Figure 4 that the overall error rate increased from Day 4 to Day 5 by 0.28 , in line with the general trend from Days 1 to 4 , although the change was not statistically significant. Tables 2 and 3 show that the average effect of alcohol on error rate is at least as large late (1.93) as early (1.74) in practice. An additional analysis of variance was performed on the error rates to compare these effects, with length of experiment (2 days or 5 days) and alcohol group (NA or A on Day 1/Day 4 and A or NA on Day 2/Day 5) as betweensubjects factors and day (1/4 or 2/5), mapping condition, and target presence as within-subjects factors. Again, the alcohol group $\times$ day interaction was highly significant $[F(1,76)=34.68, p<.01]$ whereas the three-way interaction involving length of experiment was not $(F<1)$. It is interesting to note that there was no significant effect of length of experiment on error rate $[F(1,76)=2.57$, $p>$.1]. Since the effect of practice in the 5-day experiment was to increase speed but decrease accuracy, it could have been argued that there was no evidence of improvement in visual search performance, but merely a shift in the tradeoff between speed and accuracy. However, the 5 -day subjects on Days 4 and 5 were clearly performing better than the 2-day subjects, since, as indicated, there was no difference in error rate, but a large difference of $156 \mathrm{msec}$ in speed.

To summarize the main results of the visual search task, it is clear that alcohol has no effect on speed, but impairs accuracy, the degree of impairment being unaffected by practice and task difficulty.

\section{CONCLUSIONS}

For both word categorization and visual search, alcohol had no effect on speed, but significantly impaired accuracy. This is consistent with Maylor, Rabbitt, Sahgal, and Wright (1987), in which it was argued that a dose of alcohol similar to that used in the present study adversely affects speed but has little influence on error rate for tasks in which subjects are aware of most of their errors (e.g., choice reaction time), whereas the opposite is true (increased error rate, but little effect on speed) for tasks (such as visual search) in which subjects tend not to be aware of making errors (i.e., in terms of both missed targets and false positives).

From the data, it is possible to answer the questions raised in the introduction. Although some of the conclusions are unexpected, they are all unambiguously supported by the results of the two experiments. First, improvement in performance with practice does not lead to a corresponding reduction in the amount of impairment caused by alcohol. Second, we can find no evidence for state-specific practice, tolerance, or different rates of learning with and without alcohol. For example, subjects who have practiced with alcohol still perform better in the final session without alcohol. Furthermore, their performance when alcohol is removed is at least as good as the performance of the group that practiced without alcohol for an equivalent period of time. Third, there is no empirical support for the suggestion made by Schneider, Dumais, and Shiffrin (1984) that "any physiological or psychological effects that reduce capacity should primarily affect the performance of control processes and have only a minor effect on automatic processing" (p. 15). Rather, in the present study, degree of automaticity of performance, whether defined by type of attentional demand, level of practice, or the interaction between these two variables, did not modify the amount of impairment caused by alcohol. That is, the effect of alcohol was similar in $\mathrm{CM}$ and VM conditions, early and late in practice, both for word categorization (in which practice over the course of the experiment rendered the CM task increasingly automatic) and visual search (in which prior experience of letter and digit categories ensured that the CM task could be performed more automatically than the VM task from the start of the experiment).

It could be argued that these conclusions are somewhat limited by the small amounts of practice, or that the CM and VM conditions did not differ sufficiently in terms of attentional requirements. On the question of practice, 200 trials per day in the visual search task resulted in a reduction in mean reaction time from approximately $800 \mathrm{msec}$ to just over $500 \mathrm{msec}$ from Day 1 to Day 5 . Regarding the second point, the CM and VM tasks may not represent the extreme ends of the automatic-effortful attentional continuum. But they are sufficiently separated to produce substantial differences in both speed and accuracy. For the visual search task, the average mean reaction time for CM was $550 \mathrm{msec}$, while that for VM was over $700 \mathrm{msec}$. And VM was at least $50 \%$ more error-prone than CM. Finally, it may be argued that the particular dose of alcohol used in the study was too small to produce much effect on performance. The dose was indeed small, but the effects on performance (increases in error rate of 
almost 50\%) were highly significant. In any case, the use of a low dose may be regarded as a very sensitive test of the hypotheses investigated in the present study, that, for example, practiced or automatic performance is alcohol-resistant whereas unpracticed or controlled performance is not. One would expect a huge dose of alcohol to produce gross impairments, overwhelming possible differences between tasks and conditions. The production of identical, small changes with a low dose is a far more stringent test of the claims.

\section{REFERENCES}

Battig, W. F., Montague, W. E. (1969). Category norms for verbal items in 56 categories: A replication and extension of the Connecticut category norms. Joumal of Experimental Psychology Monographs, 80 (3, Pt. II), 1-46.

Drew, G. C., Colquhoun, W. P., Long, H. A. (1959). Effects of small doses of alcohol on a skill resembling driving (MRC Memorandum No. 38). London: Her Majesty's Stationery Office.

FISK, A. D., SCHNEIDER, W. (1982). Type of task practice and timesharing activities predict performance deficits due to alcohol ingestion. Paper presented to the Human Factors Society, Santa Monica, California.

HASHER, L., \&ACKS, R. T. (1979). Automatic and effortful processes in memory. Journal of Experimental Psychology: General, 108, 356-388.

HunTLEy, M. S. (1973). Alcohol influences upon closed-course driving performance. Journal of Safeiy Research, 5, 149-164.

Kahneman, D. (1973). Attention and effort. Englewood Cliffs, NJ: Prentice-Hall.
Lewis, E. G., Dustman, R. E., Beck, E. C. (1969). The effect of alcohol on sensory phenomena and cognitive and motor tasks. Quarterly Journal of Studies on Alcohol, 30, 618-633.

Maylor, E. A., Rabitt, P. M. A. (1987a). Effect of alcohol on rate of forgetting. Psychopharmacology, 91, 230-235.

MAYLOR, E. A., RABBIT, P. M. A. (1987b). Effects of practice and alcohol on performance of a perceptual-motor task. Quarterly Journal of Experimental Psychology, 39a, 777-795.

Maylor, E. A., Rabbitt, P. M. A., Sahgal, A., \& Wright, C. (1987). Effects of alcohol on speed and accuracy in choice reaction time and visual search. Acta Psychologica, 65, 147-163.

Norman, D. A. (1976). Memory and attention: An introduction to human information processing (2nd ed.). New York: Wiley.

Posner, M. I. (1978). Chronometric explorations of mind. Hillsdale, NJ: Erlbaum.

Poulos, C. X., Wolff, L., Zilm, D. H., Kaplan, H., \& Cappell, H. (1981). Acquisition of tolerance to alcohol-induced memory deficit in humans. Psychopharmacology, 73, 176-179.

SChNeIDER, W., Dumais, S. T., \& Shiffrun, R. M. (1984). Automatic and control processing and attention. In R. Parasuraman \& D. R. Davies (Eds.), Varieties of attention (pp. 1-27). New York: Academic Press.

SCHNEIDER, W., \& FiSK, A. D. (1984). Automatic category search and its transfer. Journal of Experimental Psychology: Learning, Memory, \& Cognition, 10, 1-15.

SCHNEIDER, W., SHIFFrin, R. M. (1977). Controlled and automatic human information processing: I. Detection, search and attention. Psychological Review, 84, 1-66.

(Manuscript received September 18, 1987; revision accepted for publication December 2, 1987.) 